\title{
Visualizing Platinum Supraparticle Formation with Liquid Cell Electron Microscopy and Correlative Investigation of Catalytic Activity
}

Mei Wang, ${ }^{1}$ Chiwoo Park, ${ }^{2}$ Taylor J. Woehl ${ }^{1 *}$

${ }^{1 .}$ Department of Chemical and Biomolecular Engineering, University of Maryland, College Park, USA.

2. Department of Industrial and Manufacturing Engineering, Florida State University, Tallahassee, USA.

* Corresponding author: tjwoehl@umd.edu

Platinum nanomaterials have been widely used as heterogeneous catalysts for oxidative dehydrogenation of organic alkanes and catalytic reforming [1-2]. Catalytic reactions are sensitive to the size, shape, and surface structure of catalyst nanoparticles, which control selectivity and activity of catalytic reaction processes [3]. Further, mesoscopic effects, like spatial vicinity of catalyst particles and confinement, are known to greatly affect activity and selectivity [4-5]. However, the reaction kinetics and nanocrystal formation processes that control the atomic scale and mesoscale properties of catalyst nanoparticles during synthesis are not well-known due to the lack of in situ characterization methods. Liquid cell scanning transmission electron microcopy (LC-STEM) provides a way to visualize and quantify the kinetics of these processes on the single nanoparticle scale, which can provide us with new insights into synthesis that will guide developing catalysts with desired structures and enhanced activity and selectivity.

Here we show systematic control over platinum supraparticle structure by tuning the formation kinetics of platinum nanoparticles using electron beam nanochemistry [6]. Magnification and beam current of the electron beam were systematically changed during the experiments to investigate the effect of the dose rate on the morphologies of platinum supraparticles. Single particle tracking image analysis was used to measure nucleation and growth kinetics of each supraparticle during the experiment. Ex-situ highresolution transmission electron microscopy (HRTEM) was used to characterize the morphology of platinum supraparticles. Catalytic oxidation of resazurin into red fluorescent resofurin in the presence of hydrogen peroxide was used to probe the effect of platinum supraparticle structure on catalytical activity $[5]$.

Figure 1 (a-c) shows in situ bright-field LC-STEM images of the different supraparticle morphologies we observed. We can see that core-shell, dense, and dendritic nanoparticles were synthesized via different experimental conditions. The phase diagram (Figure 1d) shows that beam current was a key parameter for controlling morphologies of platinum supraparticles. A low beam current formed core-shell particles while medium beam current resulted in seemingly solid platinum nanoparticles. Dendritic particles only formed at high beam current. Ex-situ HRTEM images (Figure 1e-g) revealed that both "core-shell" particles and dense particles were made of $\sim 3 \mathrm{~nm}$ primary particles. This suggests that primary particles nucleated in solution and then rapidly aggregated into platinum supraparticles on the $\mathrm{Si}_{3} \mathrm{~N}_{4}$ membrane. Single particle tracking showed the growth of platinum supraparticles followed a power law $\mathrm{R} \sim \mathrm{t}^{a}$. Figure $1 \mathrm{~h}$ shows the median growth coefficient, $a$, for three morphologies of platinum particles. It is obvious that they have different growth behavior, which likely contributes to the different morphologies. For this dataset, coreshell, dense and dendritic particles showed the median growth rate coefficient of $0.73,0.42$, and 0.16 , respectively.

Figure 2 shows the measurement of catalytic activity of $30 \mathrm{~nm}$ commercial spherical platinum particles using fluorescence microscope. The optical image (Figure 2a) revealed that platinum clusters are present 
on the $\mathrm{Si}_{3} \mathrm{~N}_{4}$ window. The time lapse series of fluorescence images (Figure $2 \mathrm{~b}$-d) showed that the red fluorescence signal got stronger near the platinum particles as resazurin was catalytically converted to fluorescent resafurin, indicating this is a good method for testing the catalytic activity of platinum supraparticles with different morphologies formed from the LC-STEM experiments [7].

\section{References:}

[1] S. Vajda et al., Nat. Mater. 8(3) (2009), p. 213-216.

[2] R.E. Jentoft et al., J. Catal. 179(2) (1998), p. 565-580.

[3] I. Lee et al., Proc. Natl. Acad. Sci. 105(40) (2008), p. 15241-15246.

[4] H. Mistry et al., ACS Catal. 6(2) (2016), p. 1075-1080.

[5] B. Dong et al., Nat. Catal. 1(2) (2018), p. 135-140.

[6] M. Wang, C. Park and T.J. Woehl, Chem. Mater. 30(21) (2018), p. 7727-7736.

[7] M.W. acknowledges a Senger's Travel award from CHBE department at University of Maryland.
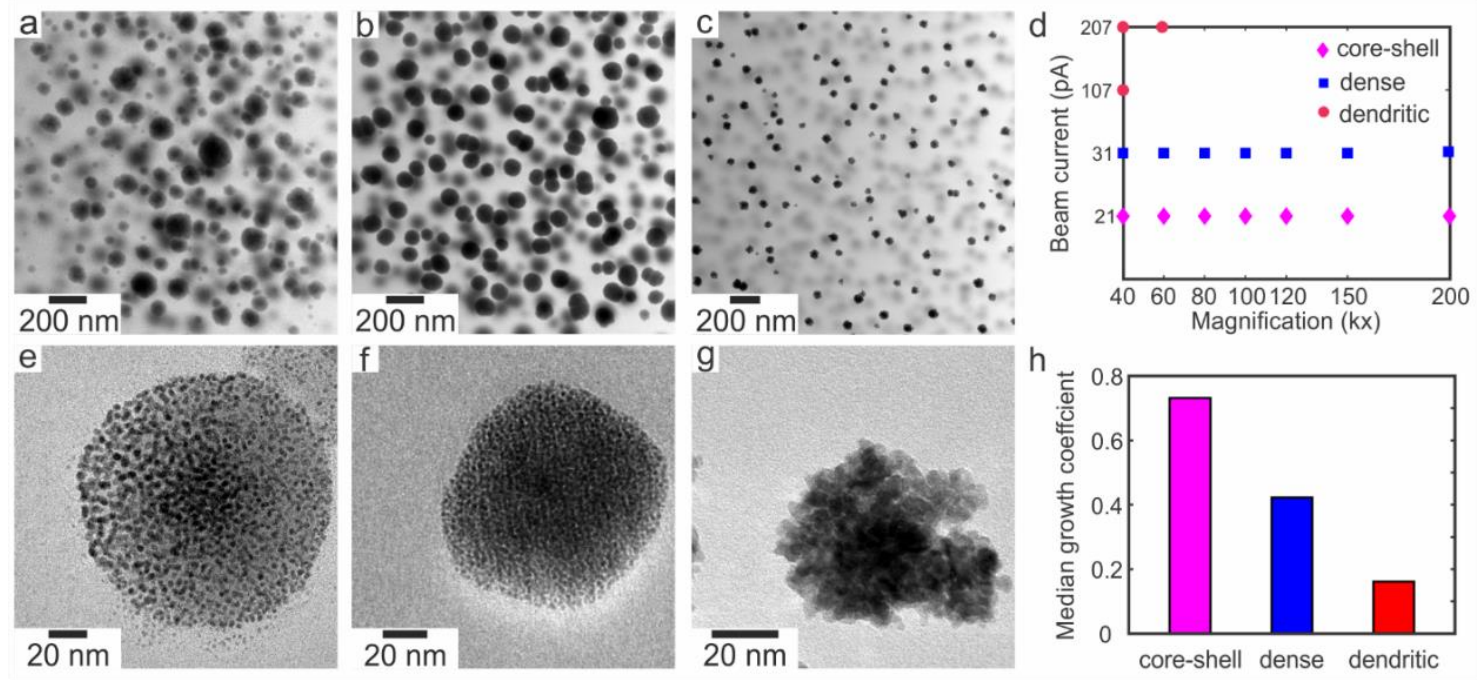

Figure 1. a-c) Examples of bright field LC-STEM images of platinum supraparticles with different morphologies, a) The magnification is $150 \mathrm{kx}$, beam current is $21 \mathrm{pA}$, electron flux is $0.447 e^{-} / \AA^{2} s$; b) The magnification is $150 \mathrm{kx}$, beam current is $31 \mathrm{pA}$, electron flux is $0.66 e^{-} / \AA^{2} s$; c) The magnification is $60 \mathrm{kx}$, beam current is $207 \mathrm{pA}$, electron flux is $0.483 e^{-} / \AA^{2} s$; d) Qualitative phase diagram of supraparticle morphology as a function of beam current and magnification. e-g) Ex-situ HRTEM images of different Pt supraparticle morphologies. h) Examples of median power law growth coefficients for coreshell, dense, and dendritic supraparticles for the data shown in Figure 1 (a-c).
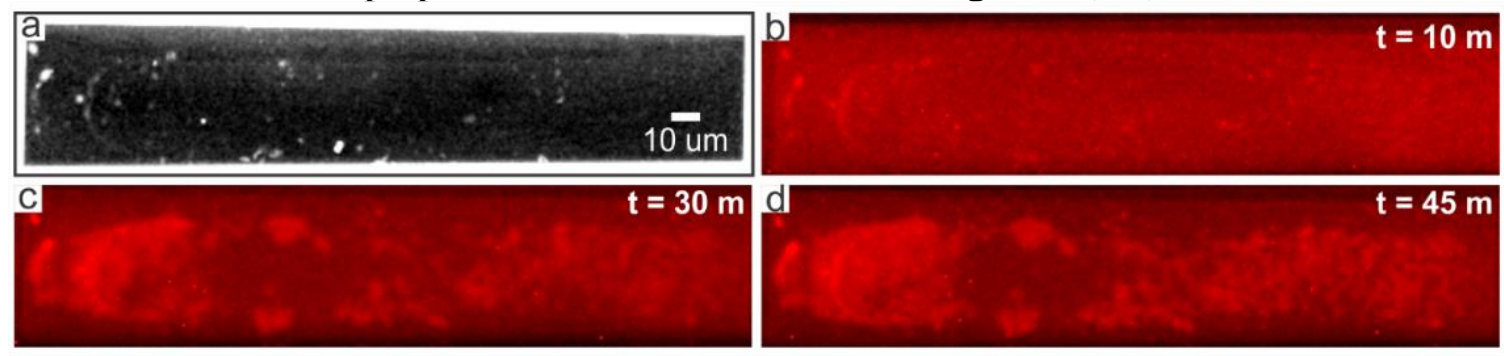

Figure 2. a) Inverted contrast bright field optical image of commercial Pt nanoparticles in the liquid cell. b-d) Time lapse series of fluorescence images of catalysis of $1 \mu \mathrm{M}$ resazurin with $20 \mathrm{mM} \mathrm{H}_{2} \mathrm{O}_{2}, \mathrm{pH}$ 7.5. 